SLAC-PUB-10753

September 2004

\title{
Pedagogical Introduction to Extra Dimensions
}

\author{
Thomas G. Rizzo \\ SLAC, Stanford, CA 94025, USA
}

\begin{abstract}
Extra dimensions provide a new window on a number of problems faced by the Standard Model. The following provides an introduction to this very broad subject aimed at experimental graduate students and post-docs based on a lecture given at the 2004 SLAC Summer Institute.
\end{abstract}

\section{Introduction: Why Study Extra Dimensions?}

Most physicists agree that some form of New Physics (NP) must exist beyond the Standard Model(SM)-we simply don't know what it is yet. Though there are many prejudices based on preconceived ideas about the form this NP may take, it will be up to experiments at future colliders, the LHC and ILC, to reveal its true nature. While we are all familiar with the list of these theoretical possibilities one must keep in mind that nature may prove to be more creative than we are and that something completely unexpected may be discovered. After all, we certainly have not yet explored more than a fraction of the theory landscape.

One now well-known possibility is that extra spatial dimensions will begin to show themselves at or near the TeV scale. Only a few years ago not many of us would have thought this even a remote possibility, yet the discovery of extra dimensions(EDs) would produce a fundamental change in how we view the universe. The study of the physics of TeV-scale EDs that has taken place over the past few years has its origins in the ground breaking work of Arkani-Hamed, Dimopoulos and Dvali(ADD)[1]. Since that time EDs has evolved from a single idea to a new paradigm with many authors employing EDs as a tool to address the large number of outstanding issues that remain unanswerable in the SM context. This in turn has lead to other phenomenological implications which should be testable at colliders and elsewhere. A (very) incomplete list of some of these ideas includes, e.g.,

- addressing the hierarchy problem[1,2]

- producing electroweak symmetry breaking without a Higgs boson[3]

- the generation of the ordinary fermion and neutrino mass hierarchy, the CKM matrix and new sources of CP violation[4]

- TeV scale grand unification or unification without SUSY while suppressing proton decay[5]

- new Dark Matter candidates and a new cosmological perspective[6, 7]

- black hole production at future colliders as a window on quantum gravity[8]

This list hardly does justice to the wide range of issues that have been considered in the ED context. Clearly a discussion of all these ideas is beyond the scope of the present work and only some of them will be briefly considered in the text which follows. However it is clear from this list that ED ideas have found their way into essentially every area of interest in high energy physics which certainly makes them worthy of detailed study.

Of course for many the real reason to study extra dimensions is that they are fun to think about and almost always lead to surprising and unanticipated results. 


\section{Thinking About Extra Dimensions}

Most analyses of EDs are within the context of quantum field theory. Can we learn anything about EDs from 'classical' considerations and some general principles without going into the complexities of field theory? Consider a massless particle moving in 5D 'Cartesian' co-ordinates and assume that 5D Lorentz invariance holds. Then the square of the $5 \mathrm{D}$ momentum for this particle is given by $p^{2}=0=g_{A B} p^{A} p^{B}=p_{0}^{2}-\mathbf{p}^{2} \pm p_{5}^{2}$ where $g_{A B}=$ $\operatorname{diag}\left(1,-1,-1,-1, \pm 1\right.$ ) is the $5 \mathrm{D}$ metric tensor (i.e., defined by the invariant interval $\left.d s^{2}=g_{A B} d x^{A} d x^{B}\right), p_{0}$ is the usual particle energy, $\mathbf{p}^{2}$ is the square of the particle 3-momentum and $p_{5}$ is its momentum along the 5 th dimension. (Note that the indices $A, B$ run over all $5 \mathrm{D}$. We will sometimes denote the 5 th dimension as $x_{5}$ and sometimes just as $y$.) The 'zero' in the equality above arises from the fact that the particle is assumed to be massless in $5 \mathrm{D}$. Note that a priori we do not know the sign of the metric tensor for the 5th dimension but as we will now see some basic physics dictates a preference; the choice of the $+(-)$ sign corresponds to either a time- or space-like ED. We can re-write the equation above in a more traditional form as $p_{0}^{2}-\mathbf{p}^{2}=p_{\mu} p^{\mu}=\mp p_{5}^{2}$ and we recall, for all the familiar particles we know of which satisfy $4 \mathrm{D}$ Lorentz invariance, that $p_{\mu} p^{\mu}=m^{2}$, which is just the square of the particle mass. (Note that Greek indices will be assumed to run only over $4 \mathrm{D}$ here.) Notice if we choose a time-like extra dimension that the sign of the square of the mass of the particle will appear to be negative, i.e., the particle is a tachyon. Tachyons are well known to be very dangerous in most models, even classically, as they can cause severe causality problems[9]-something we'd like to avoid in any theory-provided they interact with SM particles. This implies that we should pick the space-like solution. Generally, it turns out that to avoid tachyons appearing in our ED theory we must always choose EDs to be space-like and therefore we assume there will always be only one time dimension even though we could all use some extra time. ${ }^{1}$.

Now lets think about a real massless scalar field in a flat 5D-space (assuming a space-like ED!) which is a solution of the 5D Klein-Gordon equation: $\left(\partial_{A} \partial^{A}\right) \Phi=\left(\partial_{\mu} \partial^{\mu}-\partial_{y}^{2}\right) \Phi(x, y)=0$, where $y$ here represents the extra dimension. We can do a fast and dirty trick by performing something like separation of variables, e.g., take $\Phi=\sum_{n} \chi_{n}(y) \phi_{n}(x)$ and plug it into the Klein-Gordon equation above giving us $\sum_{n}\left(\chi_{n} \partial_{\mu} \partial^{\mu} \phi_{n}-\phi_{n} \partial_{y}^{2} \chi_{n}\right)=0$. Now we note that if $\partial_{y}^{2} \chi_{n}=-m_{n}^{2} \chi_{n}$, we obtain a set of equations that appears like $\sum_{n} \chi_{n}\left(\partial_{\mu} \partial^{\mu}+m_{n}^{2}\right) \phi_{n}=0$ which looks like an infinite set equations for a collection of distinct $4 \mathrm{D}$ scalar fields $\phi_{n}$ with masses $m_{n}$. This bunch of states with different masses is called a Kaluza-Klein $(\mathrm{KK})$ tower. Note that we labeled the states by a set of integers $(n)$ so that the levels are discrete; we could just as well have replaced the sum by an integral and treat $n$ as a continuous variable. The difference between these two possibilities and the link to the nature of the $5 \mathrm{D}$ space and the associated boundary conditions will be made clear below.

Now we have pulled a bit of a fast one in performing this quick and dirty analysis so let us return and do a somewhat better job; we will still assume, however, that $n$ is a distinct integer label for reasons to be clarified below. Let us start from the action (i.e., the integral of the Lagrangian) for the massless 5D scalar assuming $y_{1} \leq y \leq y_{2}$ with $y_{1,2}$ for now arbitrary:

$$
S=\int d^{4} x \int_{y_{1}}^{y_{2}} d y \frac{1}{2} \partial_{A} \Phi \partial^{A} \Phi .
$$

Now we recall $\partial_{A} \Phi \partial^{A} \Phi=\partial_{\mu} \Phi \partial^{\mu} \Phi-\partial_{y} \Phi \partial_{y} \Phi$ and substitute the decomposition $\Phi=\sum_{n} \chi_{n}(y) \phi_{n}(x)$ as above. Then the integrand of the action becomes a double sum proportional to $\sum_{n m}\left[\chi_{n} \chi_{m} \partial_{\mu} \phi_{n} \partial^{\mu} \phi_{m}-\phi_{n} \phi_{m} \partial_{y} \chi_{n} \partial_{y} \chi_{m}\right]$. This appears to be a mess but we can 'diagonalize' this equation in a few steps. First if we choose to orthonormalize the $\chi_{n}$ such that

$$
\int_{y_{1}}^{y_{2}} d y \chi_{n} \chi_{m}=\delta_{n m}
$$

\footnotetext{
${ }^{1}$ See however [10] for a discussion of time-like EDs.
} 
then the kinetic term of the $\phi_{n}$ (the first one in the bracket above) reduces to a single sum after $y$ integration becoming simply $\sum_{n} \partial_{\mu} \phi_{n} \partial^{\mu} \phi_{n}$. This is essentially just sum of the kinetic terms for a set of distinct $4 \mathrm{D}$ scalars. To handle the second term in the brackets we integrate by-parts and note that if we take the boundary conditions to be

$$
\left.\chi_{m} \partial_{y} \chi_{n}\right|_{y_{1}} ^{y_{2}}=0
$$

and also require that

$$
\partial_{y}^{2} \chi_{n}=-m_{n}^{2} \chi_{n}
$$

as above, we can integrate the entire action over $y$ and obtain an effective $4 \mathrm{D}$ theory

$$
S=\int d^{4} x \frac{1}{2} \sum_{n}\left[\partial_{\mu} \phi_{n} \partial^{\mu} \phi_{n}-m_{n}^{2} \phi_{n}^{2}\right]
$$

which is just the sum of the actions of the independent $4 \mathrm{D}$ scalars labeled by $n$ with masses $m_{n}$, i.e., the KK tower states. One sees that in this derivation it was important for the above boundary conditions(BCs) to hold in order to obtain this result. It is important to note that in fact the various masses we observe in $4 \mathrm{D}$ correspond to (apparently) quantized values of $p_{y}$ for the different $\phi_{n}$.

The fields $\chi_{n}$ can be thought of as the wave functions of the various KK states in the 5th dimension and in this simple, flat 5D scenario are simple harmonic functions: $\chi_{n}=A_{n} e^{i m_{n} y}+B_{n} e^{-i m_{n} y}$. What are the $m_{n}$ ? What are $A_{n}$ and $B_{n}$ ? To say more we must discuss BCs a bit further. In thinking about BCs in this kind of model it is good to recall one's experience with one-dimensional Quantum Mechanics(QM) that we all learned (too) many years ago. First recall the Schrödinger Equation for a free particle moving along the $x$ direction. It has the same form as Eq.4 above and since the $x$ direction is infinite, i.e., noncompact, the solution is just $\psi \sim A^{\prime} e^{i p x}+B^{\prime} e^{-i p x}$ where $p$ is the particle momentum which can take on an infinite set of continuous values. We say that in this case the momentum $p$ is not quantized and this is due to the fact that the space is noncompact. Now let us consider a slightly different problem, a particle in a box, i.e., a situation where the potential is zero for $0 \leq y \leq \pi L$ but is infinite elsewhere so that the wavefunction vanishes outside this region. Since the physical region is of a finite size it is called compact. We know that the solution inside the box takes the same general form as does the case of a free particle or $\chi_{n}$ above but it must also vanish at the boundaries. These BCs tell us $A^{\prime}$ and $B^{\prime}$ so that the solutions actually takes the form $\sim \sin n y / L$ and that the momenta are quantized, i.e., $p=n / L$ with $n=1,2, \ldots$. Clearly these two situations are completely analogous to having a 5 th dimension which is either infinite (i.e., noncompact) or finite (i.e., compact) in size. Under almost all circumstances we assume that extra dimensions are compact. For a flat 5th dimension of length $\pi L$ the analysis above tells us that the KK masses are given by $m_{n}=n / L$, i.e., the masses are large if the size of the extra dimension is small. Perhaps it is natural to think that the reason that we have not seen EDs is that they are very small and the corresponding KK states are then too massive to be produced at colliders. In fact, the observation of KK excitations is the hallmark of EDs. It is interesting to observe that there are no solutions in the 'particle in a box' example corresponding to massless particles, i.e., those with $n=0$, the so-called zero modes.

There are other sorts of BCs that can be important. In QM we also examined the case of a particle moving on a circle where angular momentum is quantized. In the $5 \mathrm{D}$ case we can imagine that the 5 th dimension is curled up into a circle, $S^{1}$-a one dimensional sphere of radius $R$-so that the points $y=-\pi R$ and $y=\pi R$ are the same, i.e., we have periodic boundary conditions. Here the KK masses are given by $m_{n}=n / R$ so that we still see the correlation between the KK masses and the inverse size of the extra dimensions but the solutions take the form of $A_{n} \cos n y / R+B_{n} \sin n y / R$ with $n=0,1,2, \ldots$ Note that here a massless mode does exist due to the periodicity of the BCs. We can change this solution slightly by imagining defining a parity operation on the interval $-\pi R \leq y \leq \pi R$ which maps $y \rightarrow-y$. There are now 2 special points on this interval, call the fixed points, which are left invariant by this $Z_{2}$ operation when combined with the translation $y \rightarrow y+2 \pi R$ and the periodicity property; these are the points $y=0, \pi R$. The eigenfunctions of our wave equation must now respect the discrete, $Z_{2}$, parity symmetry so that our solutions are either $Z_{2}$-even, $\sim \cos n y / R$ or $Z_{2}$-odd, $\sim \sin n y / R$. Note that only $Z_{2}$-even states have a zero-mode amongst them. This geometry is called $S^{1} / Z_{2}$ and is the simplest example of an orbifold, a manifold with a discrete

\section{L013}


symmetry that identifies different points in the manifold, here $y$ and $-y$. The $S^{1} / Z_{2}$ orbifold is of particular interest in model building as we will see below. By the way, we note that all of the BCs of interest to us above are such as to satisfy the conditions following from Eq.3.

So far we have seen that a 5D scalar field decomposes into a tower of $4 \mathrm{D}$ scalars when going from the $5 \mathrm{D}$ to $4 \mathrm{D}$ framework. What about other 5D fields? In a way we have encountered a somewhat similar question when we first learned Special Relativity, i.e., how do 3-vectors and scalars get embedded into 4D fields? Just as a 4-vector contains a 3-vector and a 3-scalar, one finds that, e.g., a $5 \mathrm{D}$ massless gauge field (which has 3 polarization states!) contains two KK towers, one corresponding to a 4D gauge field (with 2 polarization states) and the other to a 4D scalar field. In fact in $(4+\mathrm{n})$-dimensions a gauge field will decompose into a $4 \mathrm{D}$ gauge KK tower plus $n$ distinct scalar towers. At this point a subtlety exists. We know from our previous discussion that KK tower states above the zero mode are massive. How can there be massive 4D gauge fields with only 2 transverse polarization states? Consider for simplicity the 5D case. It turns out that we can make a gauge transformation to eliminate the scalar KK tower fields and have them 'eaten' by the gauge field-in a manner similar to the Higgs-Goldstone mechanism-becoming their longitudinal components. In a way this is a geometric Goldstone mechanism. The scalar KK tower is then identified to be just the set of Goldstone bosons eaten by the gauge fields to acquire masses. Thus in the unitary or physical gauge the massless 5D gauge field becomes a massive tower of $4 \mathrm{D}$ gauge fields. In $(4+\mathrm{n})$-dimensions only one linear combination of the scalars is eaten so that a massless $(4+n)$-dimensional gauge field produces a massive tower of $4 \mathrm{D}$ gauge fields together with $n-1$ scalar towers in the unitary gauge.

To see how this $5 \mathrm{D}$ decomposition for gauge fields works in practice consider a massless $5 \mathrm{D}$ gauge field with the 5 th dimension compactified on $S^{1} / Z_{2}$. In the first step in the KK decomposition one can show that the 2-component vector KK's are $Z_{2}$-even with $5 \mathrm{D}$ wavefunctions like $\sim \cos n y / R$, whereas the KK scalars are $Z_{2}$-odd with wavefunctions like $\sim \sin n y / R$. Note the absence of an $n=0$ scalar mode due to the $Z_{2}$ orbifold symmetry. Once we employ the KK version of the Higgs-Goldstone trick all the $n>0$ gauge KK tower fields become massive 3-component gauge fields with $m_{n}=n / R$, having eaten their scalar partners. However, the zero mode remains massless, i.e., there was no Goldstone boson for it to eat. In 4D the masslessness of the zero mode tells us that gauge invariance has not been broken. We see that orbifold BCs are useful at generating massless modes and maintaining gauge invariance. One can translate these orbifold BCs for the gauge fields into the simple relations $\partial_{y} A_{n}^{\mu}\left|=0, A_{n}^{5}\right|=0$ for all $n$ and where 'l' stands for a boundary. Interestingly the physics changes completely if we change the BCs in this case. Instead of an orbifold, consider compactifying on a line segment or interval, $0 \leq y \leq \pi R$, and taking as BCs $A_{n}^{\mu}=\partial_{y} A_{n}^{5}=0$ at $y=0$ and $\partial_{y} A_{n}^{\mu}=A_{n}^{5}=0$ at $y=\pi R$. Here one now finds that $A^{\mu} \sim \sin m_{n} y / R$ and no massless zero mode exists. After the Higgs-Goldstone trick all the 4D gauge KK tower fields become massive (leaving no remaining scalars) with $m_{n}=(n+1 / 2) / R$ implying that gauge invariance is now broken. Here we see a simple example that demonstrates that we can use BCs to break gauge symmetries. The possibility that such techniques can be successfully employed to break the symmetries of the SM without the introduction of fundamental Higgs fields has been recently extensively discussed in the literature[3].

How do other higher dimensional fields decompose? As the simplest example consider the gravitational field in 5D, represented as by symmetric tensor $h^{A B}$ which decomposes as $h^{A B} \rightarrow\left(h^{\mu \nu}, h^{\mu 5}, h^{55}\right)_{n}$ when going to 4D; as before $n$ is a KK tower index. If we compactify on $S^{1} / Z_{2}$ to keep the zero mode $h_{0}^{\mu \nu}$ massless (and which we'll identify as the ordinary graviton of General Relativity) then for $n>0$ all of the $h_{n}^{\mu 5}$ and $h_{n}^{55}$ fields get eaten to generate a massive KK graviton tower with fields that have 5 polarization states (as they should). For $n=0$ there is no $h_{0}^{\mu 5}$ to eat due to the orbifold symmetry yet a massless $h^{55}$ scalar remains in addition to the massless graviton. This field is the radion, which corresponds to an fluctuation of the size of the extra dimension, and whose vacuum value must be stabilized by other new physics to keep radius the extra dimension stable[11]. The physical spectrum is then just the radion and the graviton KK tower in this case which, as we will see below, corresponds to what happens in the RS model.

In terms of manifolds on which to compactify higher dimensional fields it is easy to imagine that as we go to higher and higher dimensions the types of manifolds and the complexity of the possible orbifold symmetries grows rapidly. Typical manifolds that are most commonly considered are torii, $T^{n}$, which are simply products of circles,

\section{L013}


and spheres, $S^{n}$.

Let us now turn to a few important representative ED models.

\section{Large Extra Dimensions}

The Large Extra Dimensions scenario of Arkani-Hamed, Dimopoulos and Dvali(ADD)[1] was proposed as a potential solution to the hierarchy problem, i.e., the question of why the (reduced) Planck scale, $\bar{M}_{P l} \simeq 2.4 \cdot 10^{18} \mathrm{GeV}$, is so much larger than the weak scale $\sim 1 \mathrm{TeV}$. ADD propose that we (and all other SM particles!) live on an assumed to be rigid 4D hypersurface (sometimes called a wall or brane). On the other hand gravity is allowed to propagate in a $(4+\mathrm{n})$-dimensional 'bulk' which is, e.g., an $n$-torus, $T^{n}$. This brane is conveniently located at the origin in the EDs, i.e., $\mathbf{y}=0$. Gauss' Law then tells us that the Planck scale we measure in $4 \mathrm{D}, \bar{M}_{P l}$, is related to the true $(4+\mathrm{n})$-dimensional fundamental scale, $M_{*}$, that appears in the higher dimensional action, via the relation

$$
\bar{M}_{P l}^{2}=V_{n} M_{*}^{n+2},
$$

where $V_{n}$ is the volume of the $n$-dimensional compactified space. $M_{*}$ (sometimes denoted as $M_{D}$ in the literature) can be thought of as the true Planck scale since it appears in the higher dimensional action which is assumed to describe General Relativity in $(4+\mathrm{n})$-dimensions. Is is possible that $M_{*}$ could be $\sim$ a few TeV thus 'eliminating' the hierarchy problem? Could we have been fooled in our extrapolation of the behavior of gravity from what we know up to the TeV scale and beyond and that gravity, becomes strong at $M_{*}$ and not at $\bar{M}_{P l}$ ? To get an idea whether this scenario can work at all we need to get some idea about the size of $V_{n}$, the volume of the compactified space. As a simple example, and the one most often considered in the literature, imagine that this space is a torus $T^{n}$ all of whose radii are equal to $R$. Then it is easy to see that $V_{n}=(2 \pi R)^{n}$; knowing $\bar{M}_{P l}$ and assuming $M_{*} \sim$ a few TeV we can estimate the value of $R$. Before we do this we need to think about how gravity behaves in EDs.

If one considers two masses separated by a distance $r$ in $(n+4)$-dimensions the force of attraction now depends on the relative magnitudes of $r$ and the compactification radius $R$. To see this first imagine $r>>R$; in this case the extra dimensions are essentially invisible and to all appearances the space looks to be $4 \mathrm{D}$. Then we know that $F_{\text {grav }} \sim 1 / r^{2}$ thanks to Newton. However, in the opposite limit $r<<R$ the effects of being in a full $(4+\mathrm{n})$-dimensional space will become obvious; we don't even realize that the ED is compactified. Either via Gauss' Law or by recalling the nature of the solution to the inhomogeneous Laplace's equation in EDs one finds that now $F_{\text {grav }} \sim 1 / r^{2+n}$. Clearly one will start to see significant deviations from Newtonian gravity once $r \sim R$ so that $R$ cannot be very large. Let's assume $n=1$; then we can solve the equation above and obtain $R \sim 10^{8} \mathrm{~m}$. This is a scale of order the Earth-Sun distance over which we know Newton's Law holds very well; thus $n=1$ is excluded. Fortunately the size of $R$ decreases rapidly as $n$ increases; for $n=2$ one obtains $R \sim 100 \mu \mathrm{m}$ which is close to the limit of current table top experimental searches[12] for deviations from Newton's Law of Gravity. These are summarized in Fig. 1 from the work of Adelberger et al.[12]. Note that these deviations from Newtonian gravity are conventionally parameterized by adding a Yukawa-type interaction of relative strength $\alpha$ and scale length $\lambda$ to the usual Newtonian potential. In the figure the deviations expected in the $n=2$ scenario are shown assuming $M_{*}=1 \mathrm{TeV}$; the bounds from the data tell us that $M_{*}>$ a few $\mathrm{TeV}$ in this case.

If $n$ is further increased $R$ becomes way too small to probe for direct deviations from the $1 / r^{2}$ Law. It is interesting to note that for $n=2$, which is already being constrained by table top measurements, $R^{-1} \sim 10^{-4} \mathrm{eV}$ telling us that we have not probed gravity directly beyond energy scales of this magnitude. This ignorance is rather amazing but it is what allows the large parameter space in which the ADD model successfully functions. From this discussion it appears that the ADD model will work as long as $n \geq 2$ with $n=2$ being somewhat close to the boundary of the excluded regime. As we will see below the naive $n=2$ case is highly disfavored by other measurements though larger values of $n$ are much more weakly constrained. How large can $n$ be? If we believe in superstring theory at high scales then we can expect that $n \leq 6$ or 7 . A priori, however, there is no reason not to consider larger values in a bottom-up approach. It is curious to note that when $n \simeq 30$ one has $M_{*} R \sim 1$ which is perhaps what we might 


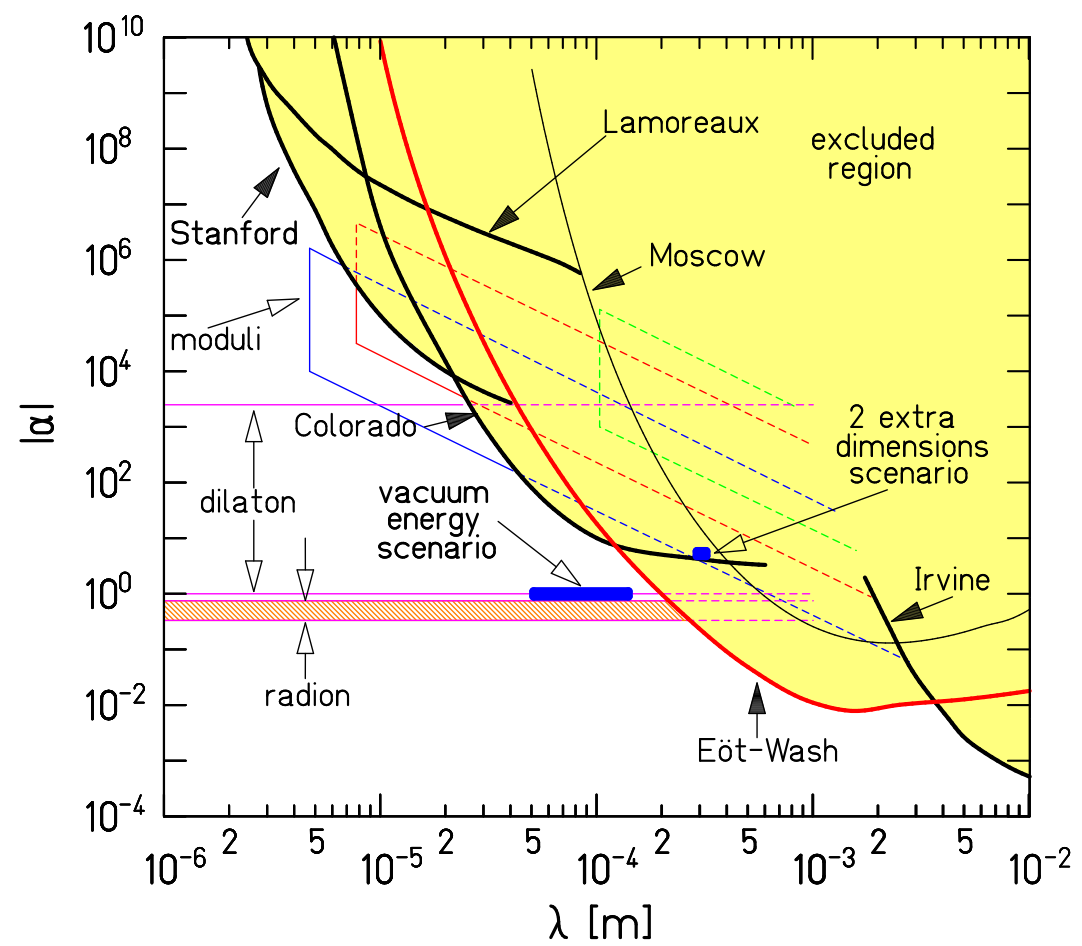

Figure 1: Regions in the $\alpha-\lambda$ plane excluded by table top searches for deviations from Newtonian gravity from Adelberger et al. [12]. The ADD prediction with $n=2$ and $M_{*}=1 \mathrm{TeV}$ is also shown.

expect based on naturalness assumptions; for any $n \sim 15$ or less, $R^{-1}<<M_{*} \sim$ a few TeV. This point is important for several reasons. (i) One may ask why we required that the SM fields remain on the brane. If the SM or any part thereof were in the bulk, those fields would have KK towers associated with them. Since the masses of these KK fields would be of order $\sim 1 / R$ as discussed above and we have not experimentally observed any KKs of the SM particles at collider so far, we must have $1 / R \geq 100 \mathrm{GeV}$ or so. For any $n \leq 10$ it is clear that this condition cannot be satisfied. So if we believe in strings the SM fields must remain on the wall. (ii) Since the gravitons are bulk fields their KK masses are given by $m_{K K}^{2}=\sum_{i=1}^{n} l_{i}^{2} / R^{2}$ where $l_{i}$ is a integer labeling the KK momenta for the $i^{\text {th }} \mathrm{ED}$. As we noted above, for not too large values of $n$ these masses are generally very small compared to the $1 \mathrm{TeV}$ or even $100 \mathrm{GeV}$ scale. This will have important phenomenological implications below.

If $n=6$ or 7 in string theory why don't we just assume this value? Consider a small variation on a theme. So far we have assumed that all of the ED compactification radii are equal; this need not be the case. Assume there are $n$ EDs but let $n-p$ of them have radius $R_{1}$ and $p$ of them radius $R_{2}$. Then from above we have

$$
\bar{M}_{P l}^{2}=(2 \pi)^{n} R_{1}^{(n-p)} R_{2}^{p} M_{*}^{(n+2)} .
$$

Now imagine that $R_{2}^{-1} \sim M_{*}$; then we'd have instead

$$
\bar{M}_{P l}^{2} \sim R_{1}^{(n-p)} M_{*}^{(n-p)+2},
$$

i.e., it would appear that we really only have $n-p$ large dimensions. The keyword here is 'large', the $p$ dimensions are actually 'small' of order $\sim \mathrm{TeV}^{-1}$ and not far from the fundamental scale in size. Thus there could be, e.g., 7 EDs as suggested by strings but only 4 of them are large. If any SM field lived only in these $p$ small EDs that would be (at least superficially) experimentally acceptable since their KK masses would be $\sim \mathrm{TeV}$ and as of yet these KKs would be unobserved at colliders. Since the SM fields can live in these TeV size EDs we will see below this the possibility is quite popular for model building purposes. 
To proceed further we need to know what these KK graviton states do, i.e., how they interact with the SM fields on the wall. A derivation of the Feynman rules for the ADD model is beyond the scope of the present talk but can be found in Ref.[13] with some elementary applications discussed in Ref.[14]. A glance at the Feynman rules tells us several things: $(i)$ all of the states in the graviton KK tower couple to SM matter on the wall with the same strength as the ordinary zero-mode graviton, i.e., to lowest order in the coupling

$$
\mathcal{L}=-\frac{1}{\bar{M}_{P l}} \sum_{n} G_{n}^{\mu \nu} T_{\mu \nu}
$$

where $G_{n}^{\mu \nu}$ are the KK graviton fields in the unitary gauge and $T_{\mu \nu}$ is the stress-energy tensor of the SM wall fields. (ii) Since there are at least 2 EDs we might expect that the vector fields (or some remaining combination of them) $G_{n}^{\mu i}$, where $i=1, \ldots, n$, would couple to the SM particles. It turns out that such couplings are absent by symmetry arguments since the SM wall resides at $\mathbf{y}=0$. (iii) Similarly we might expect that some combination of the scalar fields $G_{n}^{i j}$ to couple to the SM. Here in fact one KK tower of scalars does couple to $\sim T_{\mu}^{\mu} / \bar{M}_{P l}$. However, since $T_{\mu}^{\mu}=0$ for massless particles (except for anomalies) this coupling is rather small for most SM fields except for top quarks and massive gauge bosons. Thus, under most circumstances, these scalar contributions to various processes are rather small. (iv) Though each of the $G_{n}^{\mu \nu}$ are rather weakly coupled there are a lot of them and their density of states is closely packed. This is very important when performing sums over the graviton KK tower as we will see below.

How would ADD EDs appear at colliders? Essentially, there are two important signatures for ADD EDs and there has been an enormous amount of work on the phenomenology of the ADD model in the literature [For a review see [16]]. The first signature is the emission of graviton KK tower states during the collision of two SM particles. Consider, e.g., either the collision of $q \bar{q}$ to make a gluon or $e^{+} e^{-}$to make a photon and during either process have the SM fields emit a tower KK graviton states. Note that since each of the graviton KKs is very weakly coupled this cross section is quite small for any given KK state. Also, once emitted, the graviton interacts so weakly it will not scatter or decay in the detector and will thus appear only as missing energy. Now apart from their individual masses all graviton KK states will yield the same cross section as far as this final state is concerned, i.e., jet or photon plus missing energy; thus we should sum up all the contributions of the KK states that are kinematically accessible. For example, at an $e^{+} e^{-}$collider this means we sum over the contributions of all KK states with masses less than $\sqrt{s}$. This is a lot of states and, since these states are closely packed, we can replace the sum with an integral over the appropriate density of states. While no one KK graviton state yields a large cross section the resulting sum over so many KKs does yield a potentially large rate for either of the processes above. These rates only depends on the values of $n$ and $M_{*}$. As reviewed by Landsberg in these proceedings[17], both the Tevatron and LEPII have looked for such signatures with no luck and have placed bounds on the ADD model parameters[16]. Clearly it is up to searches at future colliders to find these signals if they exist.

Fig. 2 from Vacavant and Hinchliffe[18] shows the missing $E_{T}$ spectrum at the LHC assuming an integrated luminosity of $100 \mathrm{fb}^{-1}$ for the process $p p \rightarrow$ jet plus missing energy in the SM and the excess induced by ADD graviton emission assuming different values of $n=\delta$ and $M_{*}=M_{D}$. Once the rather large SM backgrounds are well understood this excess will be clearly visible. The more difficult question to address is whether such an excess if observed at the LHC would naturally be interpreted as arising from ADD EDs as other new physics can lead to the same apparent final state.

At the ILC, the backgrounds for the photon plus missing energy process are far simpler and better understood, essentially arising from the $\nu \bar{\nu}+\gamma$ final state. These backgrounds can be measured directly by modifying the electron (and positron) beam polarization(s) since the $W^{+} W^{-}$intermediate state gives the dominant contribution to this process. Measuring the excess event cross section at two different center of mass energies allows us to determine both $M_{*}$ and $n=\delta$ as shown in Fig. 3 from the TESLA TDR[19]. If fitting the data taken at different center of mass energies results in a poor $\chi^{2}$ using these parameters we will know that the photon plus missing energy excess is due to some other new physics source and not to ADD EDs.

These is another way to see, at least indirectly, the effect of graviton KKs in the ADD model: gravitons can be exchanged between colliding SM particles. This means that processes such as $q \bar{q} \rightarrow g g$ or $e^{+} e^{-} \rightarrow \mu^{+} \mu^{-}$can proceed

\section{L013}




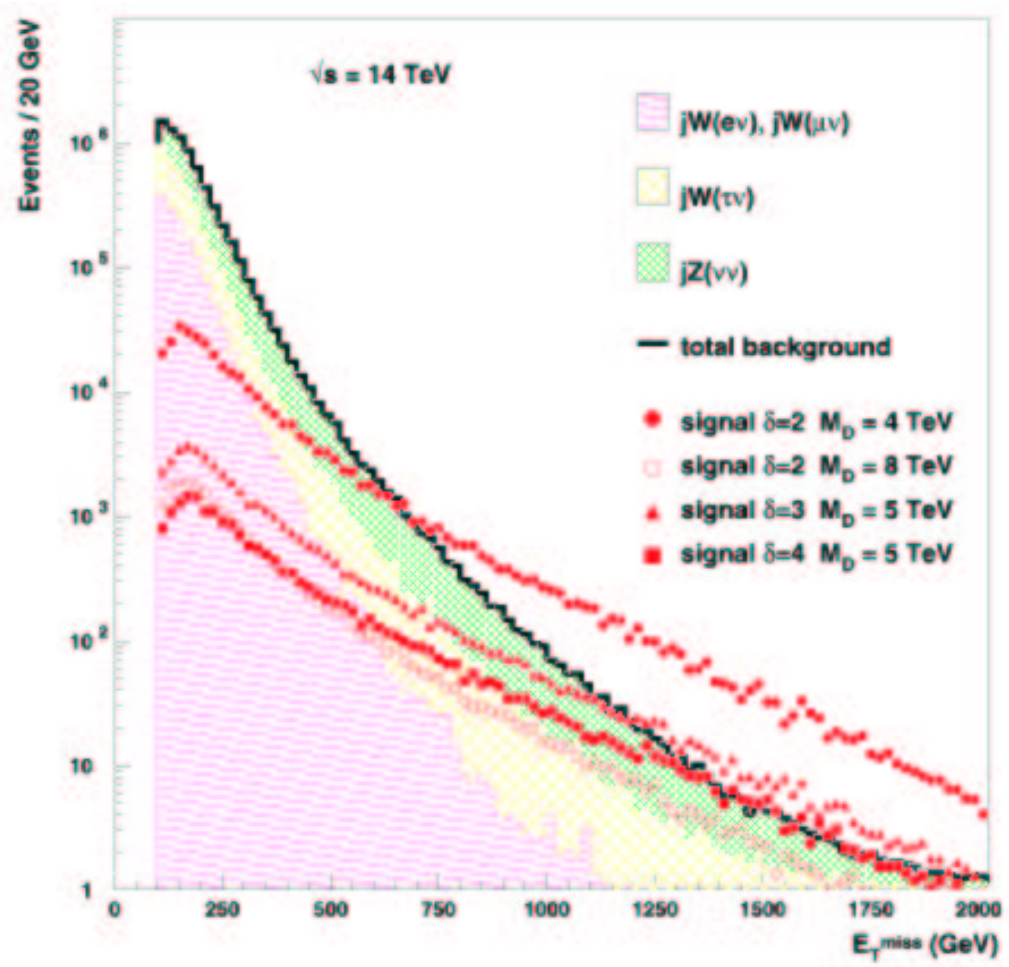

Figure 2: Missing transverse energy spectrum for the monojet plus missing $E_{T}$ signature at the LHC assuming an integrated luminosity of $100 \mathrm{fb}^{-1}$ from Ref[18]. Both the SM backgrounds and the signal excesses from graviton emission in the ADD model are shown. Here $M_{D}=M_{*}$ and $\delta=n$.

through graviton KK tower exchange as well as through the usual SM fields. As before, the amplitude for one KK intermediate state is quite tiny but we must again sum over all their exchanges (of which there are very many) thus obtaining a potentially large result. Unlike the case of graviton emission where the KK sum was cut off by the kinematics here there is no obvious cutoff and, in principle, the KK sum should include all the tower states. One problem with this is that this KK sum is divergent once $n>1$ as is the case here. (In fact the sum is log divergent for $n=2$ and power law divergent for larger $n$.) The conventional approach to this problem is to remember that once we pass the mass scale $\sim M_{*}$ the gravitons in the ADD model become strongly coupled and we can no longer rely on perturbation theory so perhaps we should cut off the sum near $M_{*}$. There are several ways to implement this in detail described in the literature[13, 14]. In all cases the effect of graviton exchange is to produce a set of dimension-8 operators containing SM fields, e.g., in the notation of Hewett[14]

$$
\mathcal{L}=\frac{4 \lambda}{\Lambda_{H}^{4}} T_{\mu \nu}^{i} T_{f}^{\mu \nu}
$$

where $\Lambda_{H} \sim M_{*}$ is the cutoff scale, $\lambda= \pm 1$ and $T_{i, f}^{\mu \nu}$ are the stress energy tensors for the SM fields in the initial and final state, respectively. This is just a contact interaction albeit of dimension- 8 and with an unconventional tensor structure owing to the spin-2 nature of the gravitons being exchanged. Graviton exchange contributions to SM processes can lead to substantial deviations from conventional expectations; Fig 4 shows the effects of graviton KK exchange on the process $e^{+} e^{-} \rightarrow b \bar{b}$ at the ILC. Note that the differential cross section as well as the left-right polarization asymmetry, $A_{L R}$, are both altered from the usual SM predictions.

Can the effects of graviton exchange be uniquely identified, i.e., separated from other new physics which induces contact interaction-like effects, such as $Z^{\prime}$ exchange? This has been addressed by several groups of authors[15]. For example, by taking moments of the $e^{+} e^{-} \rightarrow f \bar{f}, W^{+} W^{-}$angular distributions and employing polarized beams it is possible to uniquely identify the spin- 2 nature of the graviton $\mathrm{KK}$ exchange up to $\sim 6 \mathrm{TeV}$ at a $\sqrt{s}=1 \mathrm{TeV}$ ILC with an integrated luminosity of $1 \mathrm{ab}^{-1}$. This is about half of the discovery reach at the ILC for ADD EDs: 


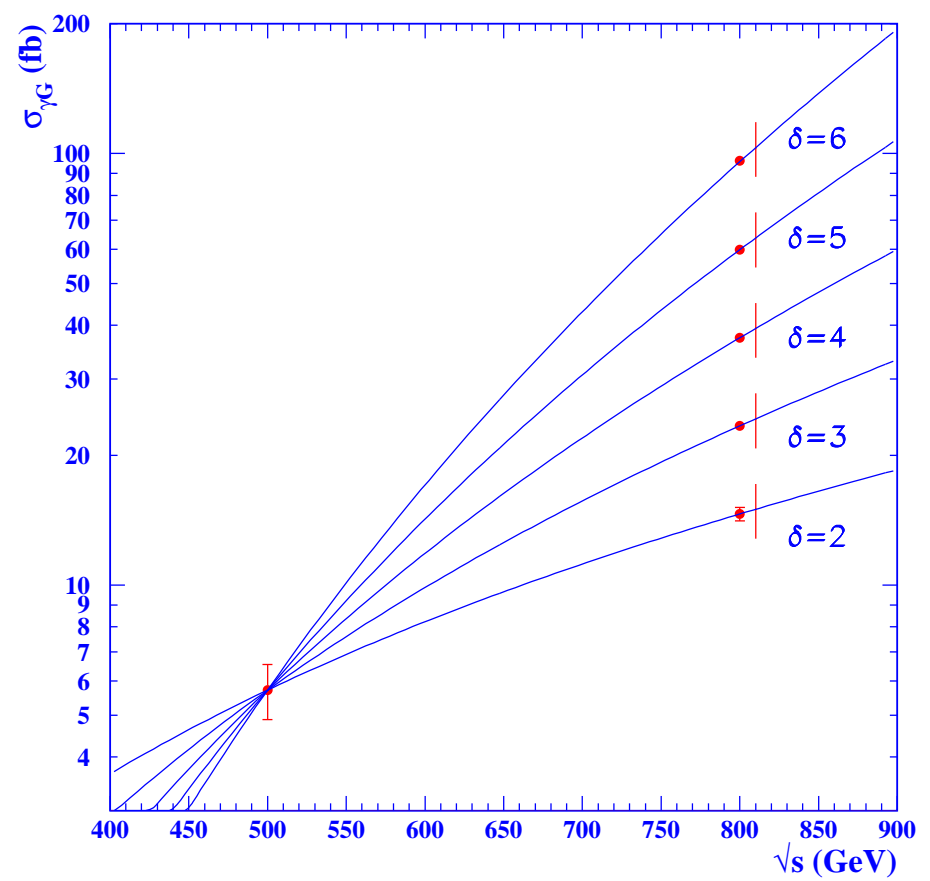

Figure 3: Signal cross section for the $\gamma$ plus missing energy final state at the ILC in the ADD model as a function of $\sqrt{s}$ for various $\delta=n$ from Ref.[19] normalized to a common value at $\sqrt{s}=500 \mathrm{GeV}$. Combining measurements at two distinct values of $\sqrt{s}$ one can extract both the values of $n$ and $M_{*}$ for the ADD model.

$\Lambda_{H} \simeq 10-11 \sqrt{s}$ for reasonable luminosities. If both beams could be polarized this could be improved somewhat by also employing transverse polarization asymmetries.

It is possible to constrain the ADD model in other ways, e.g., the emission of ADD KK gravitons can be constrained by astrophysical processes as reviewed in Ref.[16]. These essentially disfavor values of $M_{*}$ less than several hundred $\mathrm{TeV}$ for $n=2$ but yield significantly weaker bounds as $n$ increases.

Before turning to a different model let us briefly discuss the dirty little secret of the ADD model. The purpose of this model was to eliminate the hierarchy problem, i.e., remove the large ratio between the weak scale and the true fundamental scale, hence the requirement that $M_{*} \sim$ a few TeV. However, if we look carefully we see that this large ratio has been eliminated in terms of another large ratio, i.e., $R M_{*} \sim\left(\bar{M}_{P l}^{2} / M_{*}^{2}\right)^{1 / n}$, which for smallish $n$ is a very large number-as large as the hierarchy we wanted to avoid. Thus we see that ADD really only trades one large ratio for another and does not really eliminate the hierarchy problem. The next model we will discuss does a much better job in that regard.

\section{Warped Extra Dimensions}

The Warped Extra Dimensions scenario was created by Randall and Sundrum(RS)[2] and is quite different and more flexible than the ADD model. The basic RS model assumes the existence of only one ED which is compactified on the now-familiar $S^{1} / Z_{2}$ orbifold discussed above. In this setup there are two branes, one at $y=0$ (called the Planck brane) while the other is at $y=\pi r_{c}$ (called the $\mathrm{TeV}$ or SM brane) which are the two orbifold fixed points. What makes this model special is the metric:

$$
d s^{2}=e^{-2 \sigma(y)} \eta_{\mu \nu} d x^{\mu} d x^{\nu}-d y^{2},
$$


where $\eta_{\mu \nu}=\operatorname{diag}(1,-1,-1,-1)$ is the usual Minkowski metric and $\sigma(y)$ is some a priori unknown function. This type of geometry is called 'non-factorizable' because the metric of the 4D subspace is $y$-dependent. In the simplest version of the RS model it is assumed, like in the ADD case, that the SM fields live on the so-called TeV brane while gravity lives everywhere. Unlike in the ADD case, however, there is a 'cosmological' constant in the 5D bulk and both branes have distinct tensions. Solving the 5D Einstein's equations provides a unique solution for these quantities and also determines that $\sigma=k|y|$, where $k$ is a dimensionful parameter. A basic assumption of this model is that there are no large mass hierarchies present so that very roughly we expect that $k \sim M_{*}$, the $5 \mathrm{D}$ fundamental or Planck scale. In fact, once we solve Einstein's equations and plug the solutions back into the original action and integrate over $y$ we find that

$$
\bar{M}_{P l}^{2}=\frac{M_{*}^{3}}{k}\left(1-e^{-2 \pi k r_{c}}\right) .
$$

As we will see below the warp factor $e^{-\pi k r_{c}}$ will be a very small quantity which implies that $\bar{M}_{P l}, M_{*}$ and $k$ have essentially comparable magnitudes following from the assumption that no hierarchies exist. If we calculate the Ricci curvature invariant for this $5 \mathrm{D}$ space we find it is a constant, i.e., $R_{5}=-20 k^{2}$ and thus $k$ is a measure of the constant curvature of this space. A space with constant negative curvature is called an Anti-DeSitter space and so this $5 \mathrm{D}$ version is called $A d S_{5}$. Due to the presence of the exponential warp factor this space is also called a warped space. On the other hand, a space with a constant metric is called 'flat'; the ADD model is an example of flat EDs when compactified on $T^{n}$ as has been assumed here. (The $T^{n}$ spaces are flat since one can make a conformal transformation to a metric with constant coefficients.) Before going further we note that if the scale of curvature is too small, e.g., if the inverse radius of curvature becomes larger than the 5D Planck scale, then quantum gravity effects can dominate our discussion and our whole scenario may break down since we are studying the model in its 'classical', i.e., non-quantum limit. This essentially means that we must require $\left|R_{5}\right| \leq M_{*}^{2}$ which implies a bound that $k / \bar{M}_{P l} \leq 0.1$ or so, which is not much of a hierarchy.

Now for the magic of the RS model. In fitting in with the RS philosophy it will be assumed that all dimensionful parameters in the action will have their mass scale set by $M_{*} \sim \bar{M}_{P l} \sim k$ so that there is no fine-tuning. However, the warp factor rescales them as one moves about in $y$ so that, in particular, all masses will appear to be of order the $\mathrm{TeV}$ scale on the SM brane, i.e., to us. This means that if there is some mass parameter, $m$, in the action which is order $\bar{M}_{P l}$, we on TeV brane will measure it to be reduced by the warp factor, i.e., $m e^{-\pi k r_{c}}$. Note that if $k r_{c} \sim 11$ (a small hierarchy) this exponential suppression reduces a mass of order $10^{18} \mathrm{GeV}$ to only $1 \mathrm{TeV}$. Thus the ratio of the weak scale to $\bar{M}_{P l}$ is explained through an exponential factor and no large ratios appear anywhere else in the model. It has been shown by Goldberger and Wise[11] that values of $k r_{c} \sim 11$ are indeed natural and can be provided by a stable configuration. Hence we have obtained a true solution to the hierarchy problem.

How does this 'warping' really work? Let's do a simple example by considering the action for the Higgs field on the TeV brane:

$$
S=\int d^{4} x d y \sqrt{-g}\left(g^{\mu \nu} \partial_{\mu} H^{\dagger} \partial_{\nu} H-\lambda\left(H^{2}-v_{0}^{2}\right)^{2}\right) \delta\left(y-\pi r_{c}\right)
$$

where $g$ is the determinant of the metric tensor, $\lambda$ is the usual quartic coupling and $v_{0}$ is the Higgs vev, which, keeping with the RS philosophy, is assumed to be of order $\bar{M}_{P l}$. Now $\sqrt{-g}=e^{-4 k|y|}$ and $g^{\mu \nu}=e^{2 k|y|} \eta^{\mu \nu}$ so that we can trivially integrate over $y$ due to the delta-function. This yields

$$
S=\int d^{4} x\left(e^{-2 \pi k r_{c}} \eta^{\mu \nu} \partial_{\mu} H^{\dagger} \partial_{\nu} H-\lambda e^{-4 \pi k r_{c}}\left(H^{2}-v_{0}^{2}\right)^{2}\right) .
$$

Now to get a canonically normalized Higgs field (one with no extra constants in front of the kinetic term) we rescale the field by letting $H \rightarrow e^{\pi k r_{c}} h$ which now gives

$$
S=\int d^{4} x\left(\partial^{\mu} h^{\dagger} \partial_{\mu} h-\lambda\left(h^{2}-v_{0}^{2} e^{-2 \pi k r_{c}}\right)^{2}\right),
$$

\section{L013}


where we have contracted the indices using $\eta^{\mu \nu}$. We see that the vev that we observe on the SM brane is not $v_{0}$ but $v_{0} e^{-\pi k r_{c}}$ which is of order the TeV scale. Thus the Higgs gets a TeV scale vev even though the parameters we started with in the action are all of order $\bar{M}_{P l}$ ! This warping effect is a general result of the RS model.

What do the gravitons look like in this model? Even though gravitons are spin-2 it turns out that their masses and wave functions are identical to the case of a scalar field in the RS bulk[20] which is far simpler to analyze. Let us return to the Klein-Gordon equation above but now in the case of curved space; one obtains

$$
(\sqrt{-g})^{-1} \partial_{A}\left(\sqrt{-g} g^{A B} \partial_{B} \Phi\right)=0 .
$$

'Separation of variables' via the KK decomposition then yields

$$
-e^{2 k y} \partial_{y}\left(e^{-2 k y} \partial_{y} \chi_{n}\right)=m_{n}^{2} \chi_{n}
$$

which reduces to the result above for a space of zero constant curvature, i.e., when $k \rightarrow 0$. The solutions to this equation for the $\chi_{n}$ wave functions yield linear combinations of the $J_{2}, Y_{2}$ Bessel functions and not sines and cosines as in the flat space case and the masses of the KK states are given by

$$
m_{n}=x_{n} k e^{-\pi k r_{c}}
$$

where the $x_{n}$ are roots of $J_{1}\left(x_{n}\right)=0$. Here $x_{n}=0,3.8317 . ., 7.0155 . ., 10.173 . ., .$. etc. Since $k e^{-\pi k r_{c}}$ is $\sim$ a few hundred $\mathrm{GeV}$ at most, we see that the KK graviton masses are of a similar magnitude with comparable, but unequal, spacing, i.e., the KK gravitons have approximately weak/TeV scale masses. This is quite different than in the ADD model. Returning to the 5D Einstein action we can insert the wavefunctions for the KK states and determine how they couple to SM fields on the $\mathrm{TeV}$ brane; one finds

$$
\mathcal{L}=-\left(\frac{G_{0}^{\mu \nu}}{\bar{M}_{P l}}+\sum_{n>0} \frac{G_{n}^{\mu \nu}}{\Lambda_{\pi}}\right) T_{\mu \nu},
$$

where $\Lambda_{\pi}=\bar{M}_{P l} e^{-\pi k r_{c}}$ is of order TeV. Here we see that the ordinary graviton zero mode couples as in the ADD model as it should but all the higher KK modes have couplings that are exponentially larger due to the common warp factor. We thus have weak scale graviton KKs with weak scale couplings that should be produced as spin-2 resonances at colliders. Due to the universality of gravity these KK graviton resonances should be observable in many processes. There are no table top or astrophysical constraints on this scenario unlike in the ADD model. It is interesting to note that this model also has only 2 free parameters which we can take to be the mass of the lightest KK excitation, $m_{1}$, and the ratio $k / \bar{M}_{P l}$; given these parameters as input all other masses and couplings can be determined. As we will see $k / \bar{M}_{P l}$ essentially controls the KK resonance width for a fixed value of the resonance mass. The RS model in its simplest form is thus highly predictive.

At this point one may wonder why in the RS model the zero-mode coupling is so weak while the couplings of all the other KK tower states are so much stronger. The strength of the graviton KK coupling to any SM state on the $\mathrm{TeV}$ brane is proportional to the value of its $5 \mathrm{D}$ wavefunction at $y=\pi r_{c}$. In the flat space cases discussed above the typical wavefunctions for KK gravitons were $\sim \cos n y / R$ and so took on essentially the same value at the location of the SM fields for all $n$. Here the relevant combinations of the $J_{2}, Y_{2}$ Bessel functions behave quite differently when $x_{n}=0$, i.e., for the zero mode, versus the case when $x_{n}$ takes on a non-zero value as it does for the KK excitations. For the zero mode the 5D wavefunction is highly peaked near the Planck brane and so its value is very small near the $\mathrm{TeV}$ brane where we are; the opposite is true for the other KK states. Thus it is the strong peaking of the graviton wavefunctons that determine the strength of the gravitational interactions of the KK states with us.

What will these graviton KK states look like at a collider? Fig. 5 shows the production of graviton resonances at the LHC in the Drell-Yan channel and in $\mu$-pairs at the ILC for different values of $m_{1}$ and $k / \bar{M}_{P l}[20,21]$. Note that the width of the resonance grows as $\sim\left(k / \bar{M}_{P l}\right)^{2}$ so that the resonance appears rather like a spike when this ratio is small. Also note that due to the nonrenormalizable coupling of the graviton KK states the resonance width also

\section{L013}


grows as $\Gamma \sim m_{n}^{3}$ as we go up the KK tower. Hence heavier states are rather wide; for any reasonable fixed value of $k / \bar{M}_{P l}$ at some point as one goes up the KK tower one reaches states which are quite wide with $\Gamma \simeq m_{n}$ signaling the existence of the strongly interaction sector of the theory. Examining the $k / \bar{M}_{P l}-m_{1}$ parameter space and making some simple assumptions one sees that the LHC has a very good chance of covering all of it once $100 \mathrm{fb}^{-1}$ of integrated luminosity have been accumulated. Part of the present constraints on RS follow from the requirement that $k / \bar{M}_{P l} \leq 0.1$, as discussed above, and how large we are willing to let $\Lambda_{\pi}$ be before we start worrying about fine tuning again. Given these considerations we see that the LHC has excellent RS parameter space coverage as seen in Fig. 6 .

Once we discover a new resonance at the LHC or ILC we'd like to know whether or not it is a graviton KK state. The first thing to do is to determine the spin of the state; Fig. 7 shows that differentiating spin-2 from other possibilities is relatively straightforward[21, 22] at either machine. To truly identify these spin-2 resonances as gravitons, however, we need to demonstrate that they couple universally as expected from General Relativity. The only way to do this is to measure the various branching fractions and this is most easily done at the ILC. Fig. 8 shows the expected branching fractions for a graviton KK as a function of its mass assuming only decays to SM particles with a Higgs mass of $120 \mathrm{GeV}$. One unique test [21] is based on the fact that $\Gamma\left(G_{n} \rightarrow \gamma \gamma\right)=2 \Gamma\left(G_{n} \rightarrow \ell^{+} \ell^{-}\right)$both of which can be easily measured at either collider.

Before concluding this section we should note that the simple RS model scenario is barely the tip of the iceberg and has been extended in many ways to help with various model building efforts. A few possibilities that have been considered (with limited references!) are

- Extend to 3 or more branes[23]

- Extend to 6 or more dimensions[24]

- Put the SM gauge fields and fermions in the bulk[25] with or without localized brane term interactions[26]. This is very active are of current research.

\section{Universal Extra Dimensions}

Let us return to the case where we again have one flat TeV-scale ED as we might have in ADD with different compactification radii as discussed above. Then we can imagine putting all of the SM fields into this part of the bulk so that they have KK excitations; this is the Universal Extra Dimensions(UED) scenario of Appelquist, Cheng and Dobrescu[6]. For simplicity we can think of this one ED as just $S^{1} / Z_{2}$ as we are familiar with from above. The KK masses of the various fields at tree level are then given by

$$
m_{K K}^{2}=m_{S M}^{2}+n^{2} / R^{2}
$$

where $m_{S M}$ are the various SM particle masses and $R$ is the usual compactification radius. Note that even if $R^{-1}$ is only $300-500 \mathrm{GeV}$ there will be a lot of degeneracy among the KK levels. The details of the KK mass spectrum are clearly very important for understanding the phenomenology of this model, e.g., the width for $e_{1} \rightarrow e_{0} \gamma_{1}$ is very sensitive to the details of the various masses. Thus the radiative corrections to the naive mass formula above need to be considered; these were calculated by Cheng, Matchev and Schmaltz[6] and they move the spectrum around by a substantial amount. These radiative corrections arise from two sources: $(i)$ bulk terms which are finite and $(i i)$ terms induced at the orbifold fixed points which depend logarithmically on a cutoff $\Lambda$. The size of such terms are set in the model by the assumption that this non-normalizable theory is replaced by some other new physics above the scale $\Lambda \simeq 20 R^{-1}$ so that the magnitude of the divergent terms are chosen to vanish at the cutoff. Fig. 9 shows how these radiative corrections shift the tree level spectrum in this case.

In a theory with only periodic BCs, such as one compactified on $S^{1}$, the value(s) of the momenta in the extra dimensions are conserved in any process. In the case of UED, the effect of radiative corrections inducing boundary terms at the fixed points is to violate this conservation law. Instead one finds a discrete conservation law, called 
KK-parity[6], which takes on a value $(-1)^{n}$ with $n$ being the KK level. The effect of this conservation law is quite restrictive when considering the properties of the first KK states but is useful in helping the model avoid known collider and electroweak constraints. Clearly the KKs can only be pair produced and the lightest KK particle, the LKP, is stable and can be a Dark Matter candidate[6] for $R^{-1}$ in the 500-1000 GeV range; this sounds a lot like supersymmetry with $R$-parity conservation. In fact the KK spectrum after radiative corrections looks a lot like a typical SUSY spectrum. Is it possible that the two models could be confused[6]? How can they be distinguished at a collider? Clearly a major difference between SUSY and UED is that the new heavy particles have different spins in the two cases. Determining the spin of a new particle which is pair produced at the LHC is very difficult but has been discussed[27]; this is a much simpler task at the ILC as new charged spin-0 (SUSY) and spin-1/2 (UED) states have completely different threshold behaviors as discussed in Ref [28]. Another possibility, provided $R^{-1}$ is not too large, is the single production of the $n=2$ states in the UED framework. No particles analogous to these exist in the SUSY case so if observed would uniquely point to the UED scenario.

\section{Summary and Conclusion}

The subject of EDs has become a huge research area over the last six years and we have hardly scratched the surface in the present discussion. As one can see there are at present an immense number of ideas and models floating around connected to EDs and we certainly can expect there to be many more in the future. EDs can lead to a wide range of new phenomena (Dark Matter, collider signatures, etc) that will be sought over the coming decade. Of course, only experiment can tell us if EDs have anything to do with reality and, if they do exist, what their nature may be. The discovery of EDs will certainly radically alter our view of the universe on the very small and very large scales.

\section{Acknowledgments}

The author would like to thank Hooman Davoudiasl, JoAnne Hewett, Ben Lillie, Frank Petriello and Jim Wells for collaboration on research on EDs over the last 6 years. Work supported by Department of Energy contract DE-AC02-76SF00515.

\section{References}

[1] N. Arkani-Hamed, S. Dimopoulos and G. R. Dvali, Phys. Rev. D 59, 086004 (1999) [arXiv:hep-ph/9807344] and Phys. Lett. B 429, 263 (1998) [arXiv:hep-ph/9803315]; I. Antoniadis, N. Arkani-Hamed, S. Dimopoulos and G. R. Dvali, Phys. Lett. B 436, 257 (1998) [arXiv:hep-ph/9804398].

[2] L. Randall and R. Sundrum, Phys. Rev. Lett. 83, 3370 (1999) [arXiv:hep-ph/9905221].

[3] Theres been a lot of recent work on this subject; see, for example, G. Cacciapaglia, C. Csaki, C. Grojean and J. Terning, arXiv:hep-ph/0409126 and arXiv:hep-ph/0401160; C. Csaki, C. Grojean, J. Hubisz, Y. Shirman and J. Terning, Phys. Rev. D 70, 015012 (2004) [arXiv:hep-ph/0310355]; C. Csaki, C. Grojean, L. Pilo and J. Terning, Phys. Rev. Lett. 92, 101802 (2004) [arXiv:hep-ph/0308038]; C. Csaki, C. Grojean, H. Murayama, L. Pilo and J. Terning, Phys. Rev. D 69, 055006 (2004) [arXiv:hep-ph/0305237]; Y. Nomura, JHEP 0311, 050 (2003) [arXiv:hep-ph/0309189]; J. L. Hewett, B. Lillie and T. G. Rizzo, arXiv:hep-ph/0407059. H. Davoudiasl, J. L. Hewett, B. Lillie and T. G. Rizzo, JHEP 0405, 015 (2004) [arXiv:hep-ph/0403300] and Phys. Rev. D 70, 015006 (2004) [arXiv:hep-ph/0312193]; R. Barbieri, A. Pomarol and R. Rattazzi, Phys. Lett. B 591, 141 (2004) [arXiv:hep-ph/0310285].

[4] Some sample analyses can be found in N. Arkani-Hamed, S. Dimopoulos, G. R. Dvali and J. March-Russell, Phys. Rev. D 65, 024032 (2002) [arXiv:hep-ph/9811448]. N. Arkani-Hamed, Y. Grossman and M. Schmaltz, 
Phys. Rev. D 61, 115004 (2000) [arXiv:hep-ph/9909411]; N. Arkani-Hamed and M. Schmaltz, Phys. Rev. D 61, 033005 (2000) [arXiv:hep-ph/9903417]; B. Lillie, JHEP 0312, 030 (2003) [arXiv:hep-ph/0308091]; B. Lillie and J. L. Hewett, Phys. Rev. D 68, 116002 (2003) [arXiv:hep-ph/0306193]; K. Agashe, G. Perez and A. Soni, arXiv:hep-ph/0408134.

[5] K. R. Dienes, E. Dudas and T. Gherghetta, Nucl. Phys. B 537, 47 (1999) [arXiv:hep-ph/9806292] and Phys. Lett. B 436, 55 (1998) [arXiv:hep-ph/9803466]; L. Randall and M. D. Schwartz, Phys. Rev. Lett. 88, 081801 (2002) [arXiv:hep-th/0108115] and JHEP 0111, 003 (2001) [arXiv:hep-th/0108114]; M. Carena, A. Delgado, E. Ponton, T. M. P. Tait and C. E. M. Wagner, Phys. Rev. D 68, 035010 (2003) [arXiv:hep-ph/0305188].

[6] T. Appelquist, H. C. Cheng and B. A. Dobrescu, Phys. Rev. D 64, 035002 (2001) [arXiv:hep-ph/0012100]; H. C. Cheng, K. T. Matchev and M. Schmaltz, Phys. Rev. D 66, 056006 (2002) [arXiv:hep-ph/0205314]; H. C. Cheng, K. T. Matchev and M. Schmaltz, Phys. Rev. D 66, 036005 (2002) [arXiv:hep-ph/0204342]; G. Servant and T. M. P. Tait, Nucl. Phys. B 650, 391 (2003) [arXiv:hep-ph/0206071].

[7] For an introduction, see Binetruy, C. Deffayet and D. Langlois, Nucl. Phys. B 565, 269 (2000) [arXiv:hepth/9905012].

[8] S. Dimopoulos and G. Landsberg, Phys. Rev. Lett. 87, 161602 (2001) [arXiv:hep-ph/0106295]; S. B. Giddings and S. Thomas, Phys. Rev. D 65, 056010 (2002) [arXiv:hep-ph/0106219]; For a recent update, see S. B. Giddings and V. S. Rychkov, arXiv:hep-th/0409131.

[9] E. Recami, Riv. Nuovo Cim. 9N6, 1 (1986).

[10] G. R. Dvali, G. Gabadadze and G. Senjanovic, arXiv:hep-ph/9910207.

[11] W. D. Goldberger and M. B. Wise, Phys. Lett. B 475, 275 (2000) [arXiv:hep-ph/9911457] and Phys. Rev. Lett. 83, 4922 (1999) [arXiv:hep-ph/9907447].

[12] See, for example, C. D. Hoyle, D. J. Kapner, B. R. Heckel, E. G. Adelberger, J. H. Gundlach, U. Schmidt and H. E. Swanson, Phys. Rev. D 70, 042004 (2004) [arXiv:hep-ph/0405262] and references therein.

[13] T. Han, J. D. Lykken and R. J. Zhang, Phys. Rev. D 59, 105006 (1999) [arXiv:hep-ph/9811350]; G. F. Giudice, R. Rattazzi and J. D. Wells, Nucl. Phys. B 544, 3 (1999) [arXiv:hep-ph/9811291].

[14] J. L. Hewett, Phys. Rev. Lett. 82, 4765 (1999) [arXiv:hep-ph/9811356]; E. A. Mirabelli, M. Perelstein and M. E. Peskin, Phys. Rev. Lett. 82, 2236 (1999) [arXiv:hep-ph/9811337]; T. G. Rizzo, Phys. Rev. D 59, 115010 (1999) [arXiv:hep-ph/9901209].

[15] P. Osland, A. A. Pankov and N. Paver, Phys. Rev. D 68, 015007 (2003) [arXiv:hep-ph/0304123]; T. G. Rizzo, JHEP 0210, 013 (2002) [arXiv:hep-ph/0208027], JHEP 0308, 051 (2003) [arXiv:hep-ph/0306283] and JHEP 0302, 008 (2003) [arXiv:hep-ph/0211374].

[16] J. Hewett and M. Spiropulu, Ann. Rev. Nucl. Part. Sci. 52, 397 (2002) [arXiv:hep-ph/0205106].

[17] See G.Landsberg, these proceedings.

[18] L. Vacavant and I. Hinchliffe, J. Phys. G 27, 1839 (2001).

[19] J. A. Aguilar-Saavedra et al. [ECFA/DESY LC Physics Working Group Collaboration], "TESLA Technical Design Report Part III: Physics at an e+e- Linear Collider," arXiv:hep-ph/0106315.

[20] H. Davoudiasl, J. L. Hewett and T. G. Rizzo, Phys. Rev. Lett. 84, 2080 (2000) [arXiv:hep-ph/9909255].

[21] H. Davoudiasl, J. L. Hewett and T. G. Rizzo, Phys. Rev. D 63, 075004 (2001) [arXiv:hep-ph/0006041].

[22] B. C. Allanach, K. Odagiri, M. A. Parker and B. R. Webber, JHEP 0009, 019 (2000) [arXiv:hep-ph/0006114].

[23] I. I. Kogan and G. G. Ross, Phys. Lett. B 485, 255 (2000) [arXiv:hep-th/0003074]; I. I. Kogan, S. Mouslopoulos and A. Papazoglou, Phys. Lett. B 501, 140 (2001) [arXiv:hep-th/0011141]; S. H. H. Tye and I. Wasserman, Phys. Rev. Lett. 86, 1682 (2001) [arXiv:hep-th/0006068].

[24] Z. Chacko and A. E. Nelson, Phys. Rev. D 62, 085006 (2000) [arXiv:hep-th/9912186].

[25] H. Davoudiasl, J. L. Hewett and T. G. Rizzo, Phys. Lett. B 473, 43 (2000) [arXiv:hep-ph/9911262]; A. Pomarol, Phys. Lett. B 486, 153 (2000) [arXiv:hep-ph/9911294]; T. Gherghetta and A. Pomarol, Nucl. Phys. B 586, 141 (2000) [arXiv:hep-ph/0003129]; Y. Grossman and M. Neubert, Phys. Lett. B 474, 361 (2000) [arXiv:hepph/9912408]; S. J. Huber and Q. Shafi, Phys. Lett. B 498, 256 (2001) [arXiv:hep-ph/0010195] and Phys. Rev. D 63, 045010 (2001) [arXiv:hep-ph/0005286]; J. L. Hewett, F. J. Petriello and T. G. Rizzo, JHEP 0209, 030 
(2002); R. Kitano, Phys. Lett. B 481, 39 (2000) [arXiv:hep-ph/0002279].

[26] For a discussion, see, for example M. Carena, T. M. P. Tait and C. E. M. Wagner, Acta Phys. Polon. B 33, 2355 (2002) [arXiv:hep-ph/0207056]; H. Davoudiasl, J. L. Hewett and T. G. Rizzo, Phys. Rev. D 68, 045002 (2003) [arXiv:hep-ph/0212279] and JHEP 0308, 034 (2003) [arXiv:hep-ph/0305086]; M. Carena, E. Ponton, T. M. P. Tait and C. E. M. Wagner, Phys. Rev. D 67, 096006 (2003) [arXiv:hep-ph/0212307]; F. del Aguila, M. Perez-Victoria and J. Santiago, arXiv:hep-ph/0305119 and "Bulk fields with general brane kinetic terms," JHEP 0302, 051 (2003) [arXiv:hep-th/0302023].

[27] A.J. Barr, ATLAS note ATL-PHYS-2004-017, 2004.

[28] B. C. Allanach et al. [Beyond the Standard Model Working Group Collaboration], "Les Houches 'Physics at TeV Colliders 2003' Beyond the Standard Model Working Group: Summary report,” arXiv:hep-ph/0402295. 

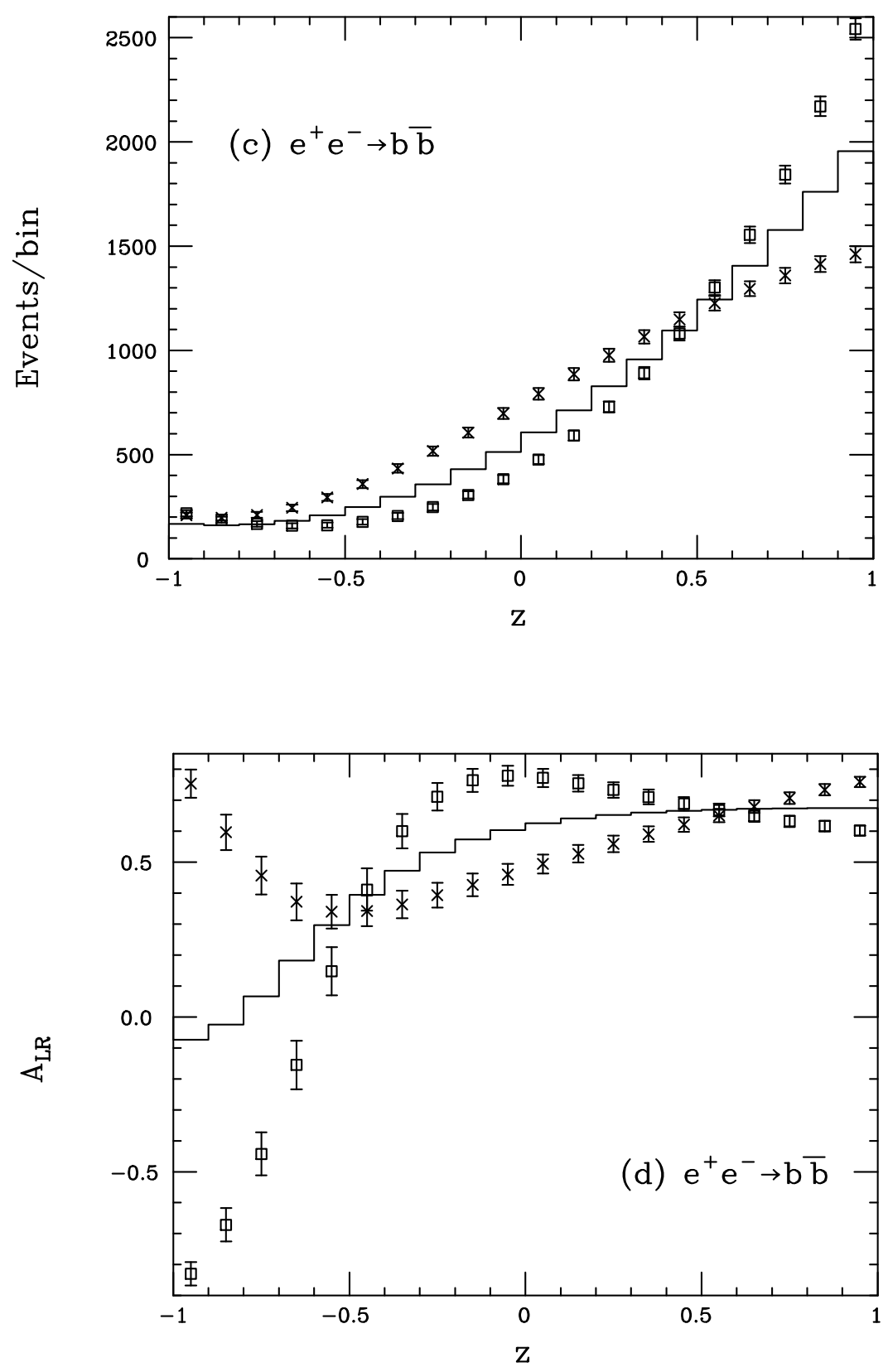

Figure 4: Deviations in the process $e^{+} e^{-} \rightarrow b \bar{b}$ at the ILC due to graviton KK tower exchange in the ADD model from Hewett[14]. The top panel is the angular distribution while the lower panel is the left-right polarization asymmetry. Here $\sqrt{s}=500 \mathrm{GeV}$ and $\Lambda_{H}=1.5 \mathrm{TeV}$. The histograms are the SM predictions while the 'data' points are for the ADD model with $\lambda= \pm 1$. An integrated luminosity of $75 \mathrm{fb}^{-1}$ has been assumed. 

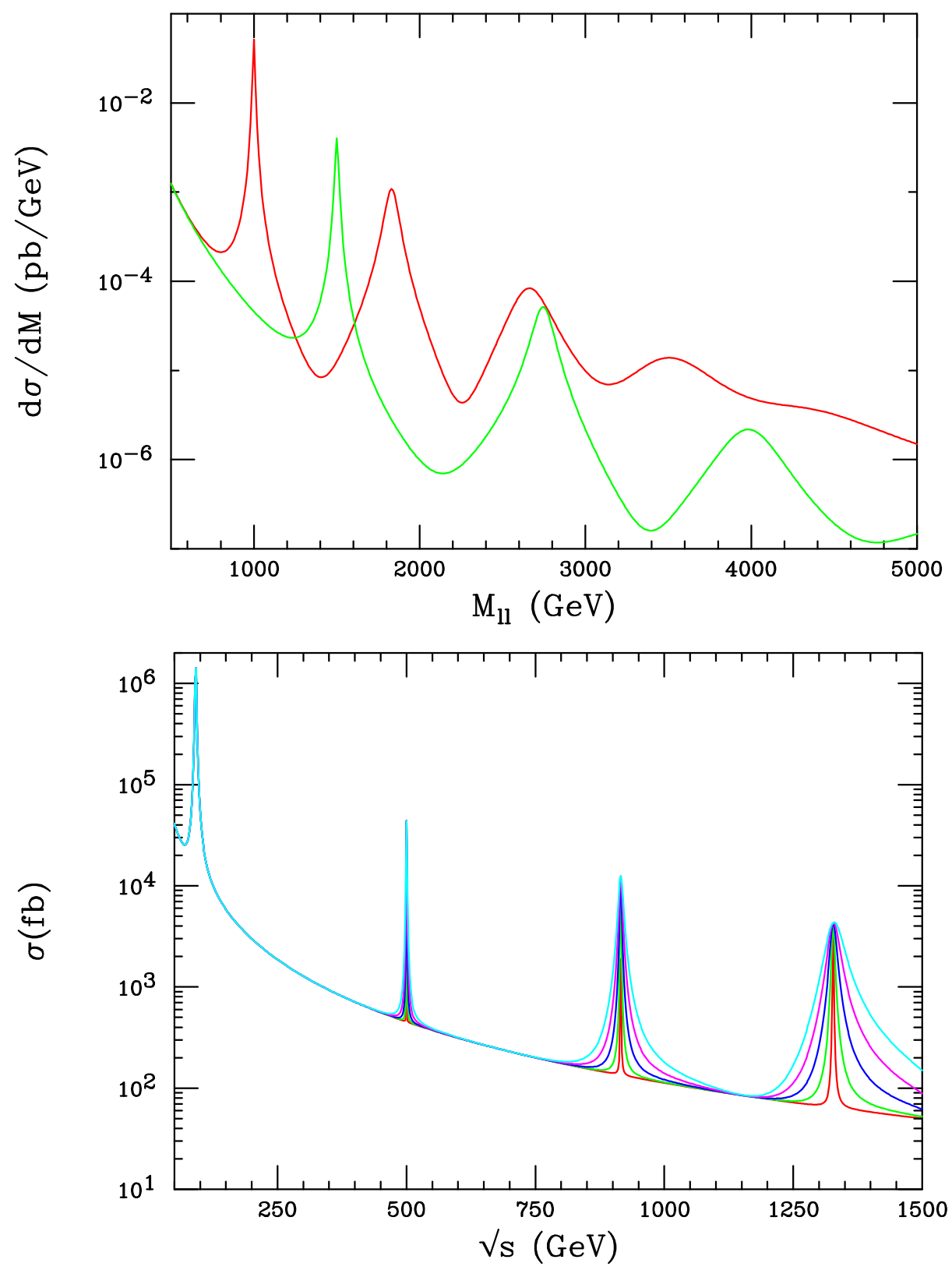

Figure 5: Graviton resonance production in Drell-Yan at the LHC(top) and at the ILC in $\mu^{+} \mu^{-}$in the RS model from Ref[21]. The different curves correspond to various choices of $k / \bar{M}_{P l}$ and $m_{1}$ as described in the text.The ever widening resonances correspond to increasing the value of $k / \bar{M}_{P l}$. 


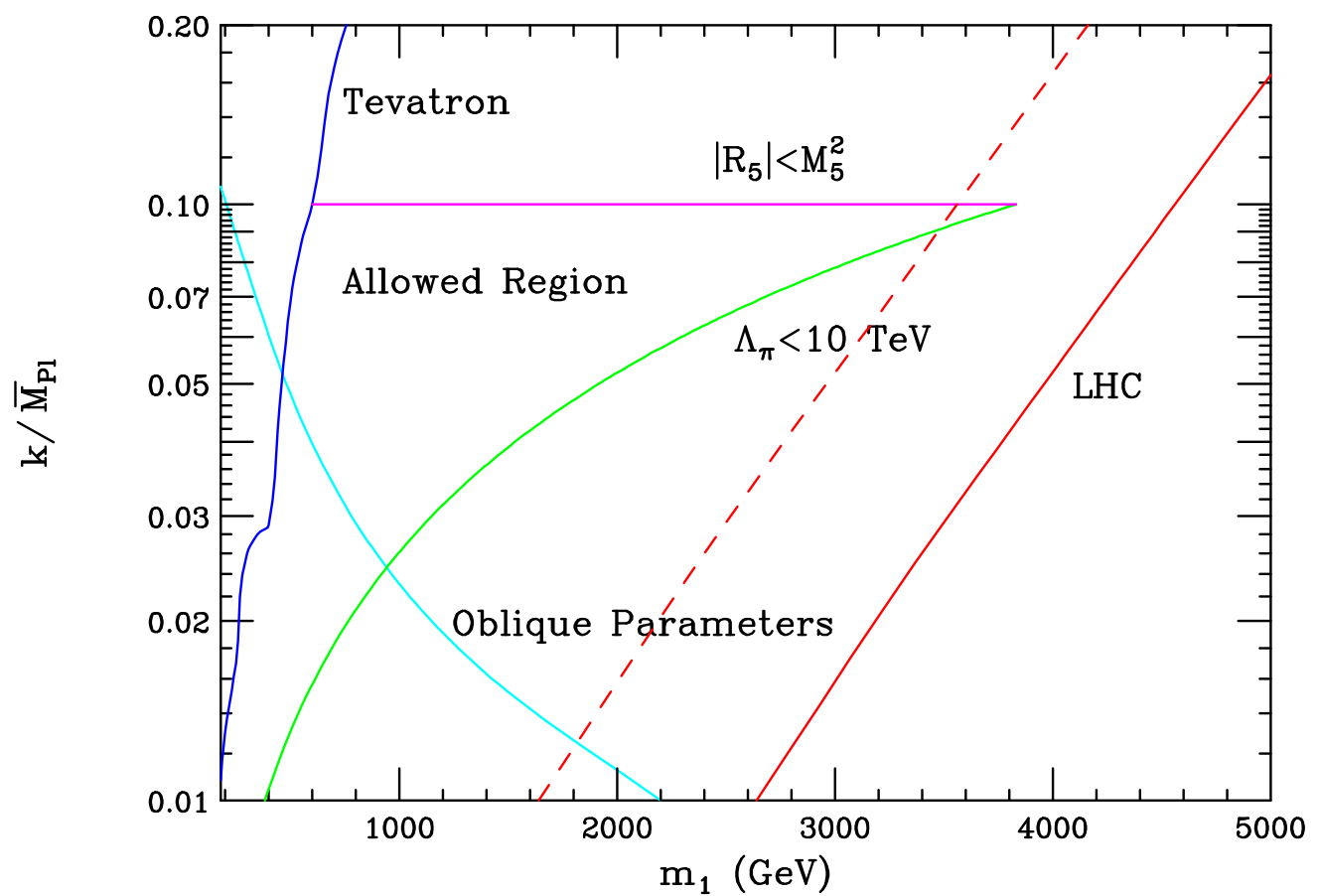

Figure 6: Allowed region in the RS model parameter space implied by various theoretical and experimental constraints from Ref.[21]. The regions to the left of the horizontal lines are excluded by direct searches at colliders. The dashed(solid) line for the LHC corresponds to an integrated luminosity of $10(100) \mathrm{fb}^{-1}$. The present anticipated parameter space is inside the triangular shaped region. 

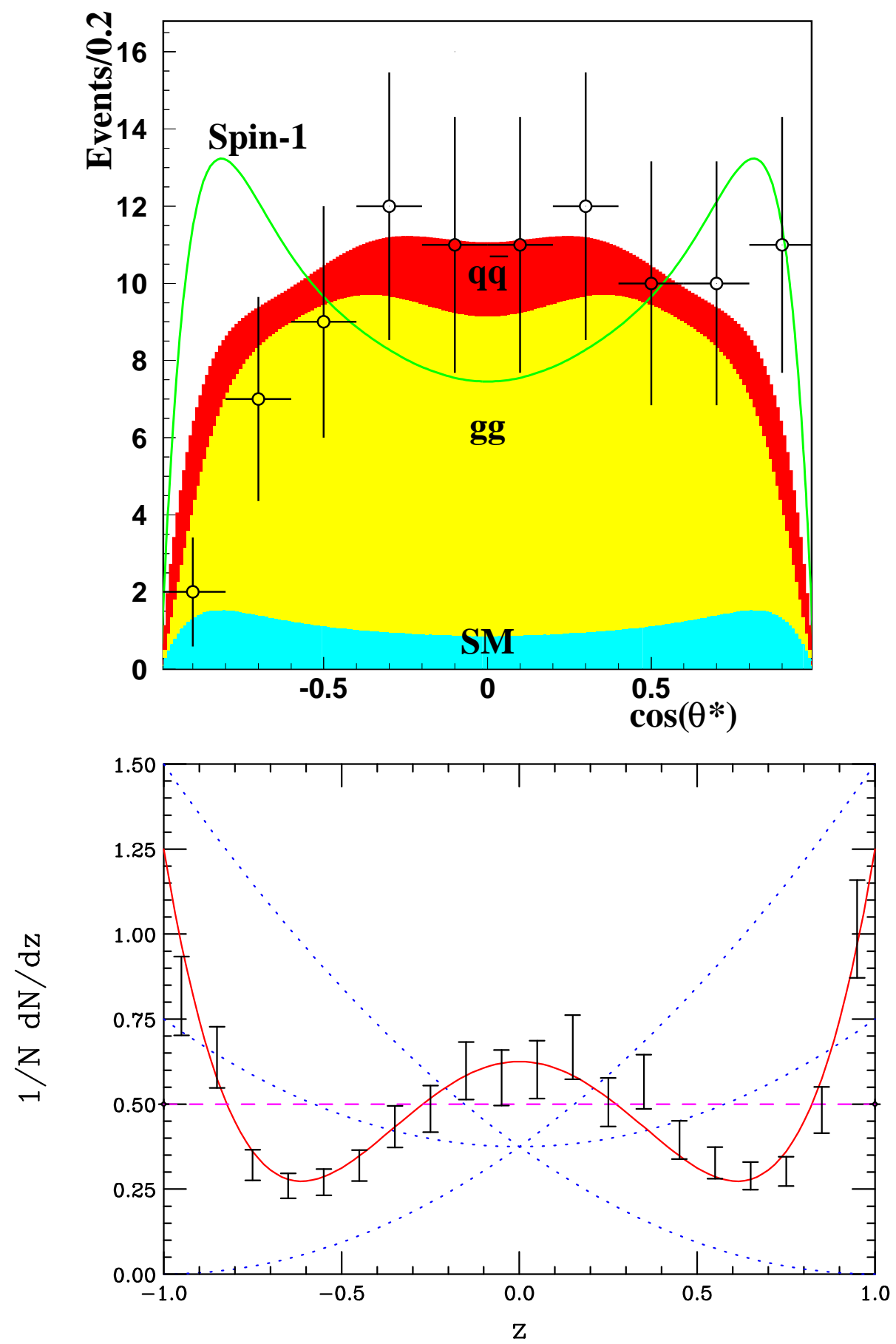

Figure 7: Results from Refs.[21, 22] showing that the spin of the KK graviton in the RS model can be determined at either the $\mathrm{LHC}$ (top) or ILC(bottom) from the angular distribution of final state dilepton pairs. Fitting the dilepton data to different spin hypotheses is relatively straightforward. 


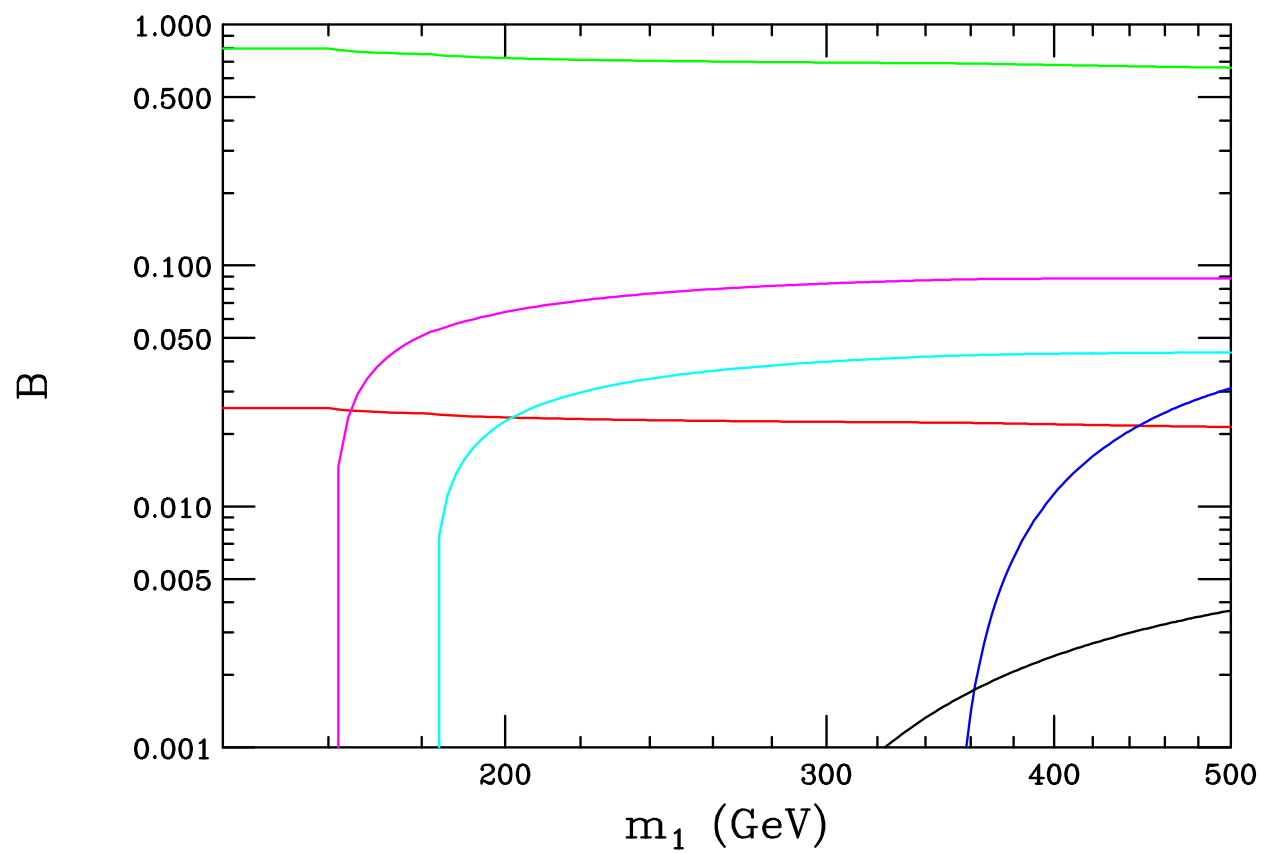

Figure 8: Branching fractions for the RS graviton KK state as a function of its mass from Ref.[21]. From top to bottom on the right hand side of the figure the curves correspond to the following final states: $j j, W^{+} W^{-}, Z Z, t \bar{t}, \ell^{+} \ell^{-}$, and $h h$, respectively.
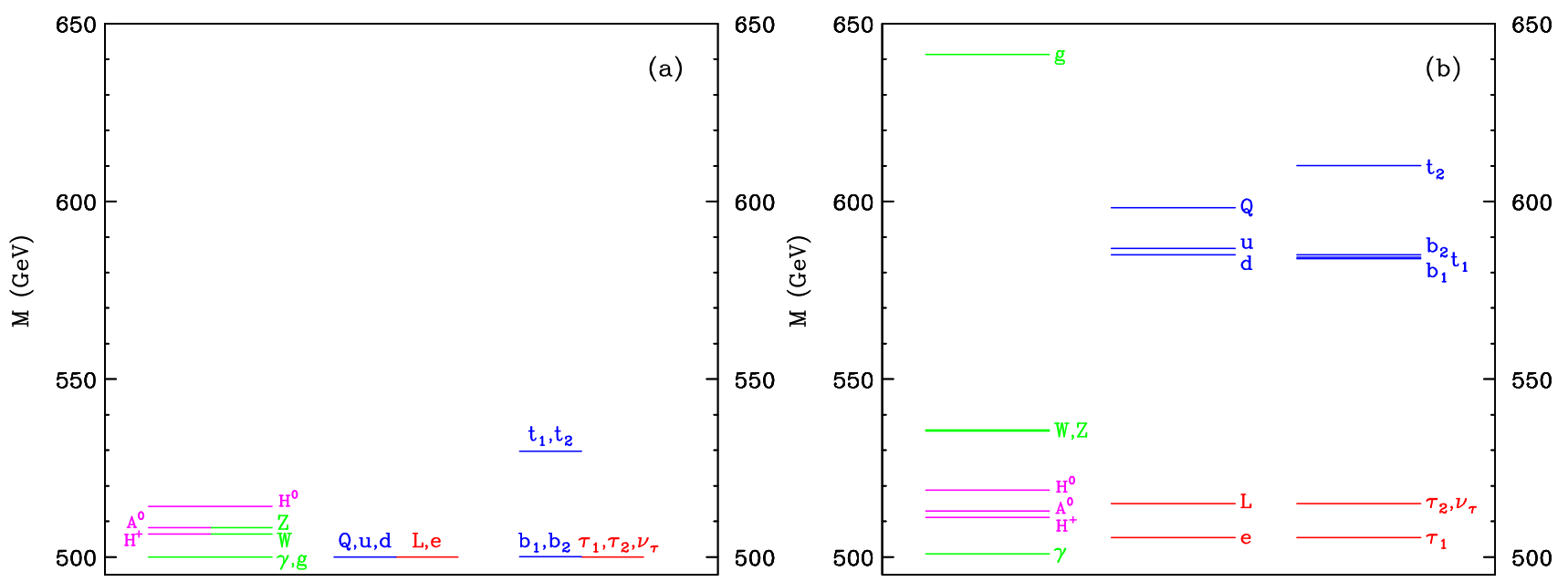

Figure 9: The spectrum of the first KK level at (a) tree level and (b) one-loop, for $R^{-1}=500 \mathrm{GeV}, \Lambda R=20, m_{h}=120$ $\mathrm{GeV}, \bar{m}_{H}^{2}=0$, and assuming vanishing boundary terms at the cut-off scale $\Lambda$ from Cheng, Matchev and Schmaltz[6]. 